\title{
Factibilidad, eficacia y satisfacción lograda por la intervención transdisciplinaria de un comité de ética clínico-hospitalario. Un estudio cualicuantitativo
}

\author{
Feasibility, effectiveness, and satisfaction achieved by the \\ transdisciplinary intervention of a clinical-hospital ethics \\ committee. A qualitative and quantitative study
}

\author{
Jorge O. Selandari $\mathbb{D}^{\mathbb{D}}$, Manuela de la Portilla ${ }^{a} \mathbb{D}$, M. Susana Ciruzzi ${ }^{a} \mathbb{D}$, \\ Claudia Couceiro $^{a}$ (D), Hernán O. García ${ }^{(\mathbb{D}}$, M. de los Ángeles Iervolino ${ }^{a}$ (D), \\ Daniela N. Marin ${ }^{a}(\mathbb{D})$, Carina Miranda ${ }^{a}(\mathbb{D})$ Luis Novali $^{a}(\mathbb{D})$, Laura Ortega ${ }^{a}$ (D), \\ Corina Ponce $^{a}$ (D), Pablo Puccara (D), Susana G. Quintana ${ }^{a}$ (D) Estela Rodríguez ${ }^{a}$ (D), \\ Fernanda Ledesma ${ }^{a} \mathbb{D}$
}

\section{RESUMEN}

Introducción. Las funciones del Comité de Ética Clínica (CEC) son educativas, normativas, consultivas, mediadoras y reflexivas. Como cualquier servicio de salud, las consultas de casos a un CEC deben ser sujetas a revisión y mejora de la calidad.

Los objetivos fueron evaluar la factibilidad, la efectividad y la satisfacción de las recomendaciones ético-asistenciales dadas por el CEC, y evaluar su impacto en el equipo tratante y en la familia.

Población y métodos. Estudio descriptivo, retrospectivo, cualicuantitativo, de casos clínicos presentados en el Comité de Ética Clínica del hospital desde el 1 de enero de 2013 hasta el 31 de diciembre de 2017, según datos del libro de actas, historias clínicas, registros de la Oficina de Comunicación a Distancia y entrevistas semiestructuradas al equipo de salud.

a. Hospital de Pediatría S.A.M.I.C. Prof. Dr. Juan P. Garrahan, Ciudad Autónoma de Buenos Aires, Argentina.

Correspondencia: Jorge O. Selandari: jselandari@intramed.net

Financiamiento: Beca de Investigación del Hospital de Pediatría S.A.M.I.C. Prof. Dr. Juan P. Garrahan.

Conflicto de intereses: Ninguno que declarar.

Recibido: 26-11-2020 Aceptado: 5-8-2021 http: / / dx.doi.org/10.5546/ aap.2022.30

Texto completo en inglés:

http: / / dx.doi.org/10.5546/ aap.2022.eng.30

Cómo citar: Selandari JO, de la Portilla M Ciruzzi MS, Couceiro C, et al. Factibilidad, eficacia y satisfacción lograda por la intervención transdisciplinaria de un comité de ética clínicohospitalario. Un estudio cualicuantitativo. Arch Argent Pediatr 2022;120(1):30-38.

\section{INTRODUCCIÓN}

Las funciones de un Comité de Ética Clínica (CEC) son variadas y pueden clasificarse como educativas, normativas, consultivas, mediadoras y reflexivas. ${ }^{1}$ Respecto de las tres últimas, se considera que, como cualquier servicio de salud, las consultas de casos individuales a un CEC deben estar sujetas a revisión y mejora de la calidad..$^{2-8}$ Sin embargo, las diferencias fundamentales con los servicios asistenciales de clínica hacen que la evaluación de la excelencia y la calidad de las consultas de ética clínica sea problemática y difícil.9-11 Es posible que por este motivo la investigación de estos aspectos sea poco frecuente.

Los objetivos fueron evaluar la factibilidad, la efectividad y la satisfacción de las recomendaciones dadas por el CEC a los problemas ético-asistenciales y, además, valorar el impacto de las recomendaciones en el equipo tratante y en la familia. 


\section{POBLACIÓN Y MÉTODOS}

Estudio descriptivo, retrospectivo y cualicuantitativo, sobre los casos clínicos presentados en el CEC del hospital desde el 1 de enero de 2013 hasta el 31 de diciembre de 2017. Se basó en datos del libro de actas, las historias clínicas (HC), registros de la Oficina de Comunicación a Distancia (OCD) y entrevistas semiestructuradas a miembros del equipo de salud que participaron en la presentación del paciente. Se excluyeron los casos en los que no se pudo obtener el documento de HC.

Los resultados de ítems de la HC que presentaban una evaluación subjetiva fueron acordados entre los investigadores y ordenados según una escala de Likert en 3 categorías (En nada, Parcialmente y Totalmente).

Una de las autoras (MDLP) realizó entrevistas al equipo tratante personalmente y por vía telefónica o por correo electrónico cuando eran pacientes consultados por otra institución.

Los ítems subjetivos de la encuesta a profesionales fueron recabados según una escala de Likert de 5 grados (ver Anexo electrónico: Formulario de encuesta y operacionalización de variables). Para facilitar su reporte, estos resultados se agruparon en tres categorías: desfavorable y favorable, como categorías extremas, y parcial, la central. Respecto de los motivos de consulta, los encuestados podían marcar, en una lista de opciones, el motivo principal, otros motivos secundarios y finalmente, mediante texto libre, podían agregar otros motivos. En los casos sin encuesta disponible, se atribuyeron los motivos principales y secundarios según el análisis de los registros.

Para facilitar la comunicación, las variables se agruparon en 3 grupos: factibilidad, efectividad y satisfacción (FES).

Se garantizó el anonimato de todos los casos evaluados y de los profesionales entrevistados mediante métodos convencionales.

El consentimiento informado fue realizado en forma oral. Para la recolección y análisis de datos, se utilizó REDCap ${ }^{\circledR}$, Excel $^{\circledR}$ y métodos estadísticos descriptivos convencionales.

El estudio fue aprobado por la Dirección de Docencia e Investigación del Hospital de Pediatría S.A.M.I.C. Prof. Dr. Juan P. Garrahan.

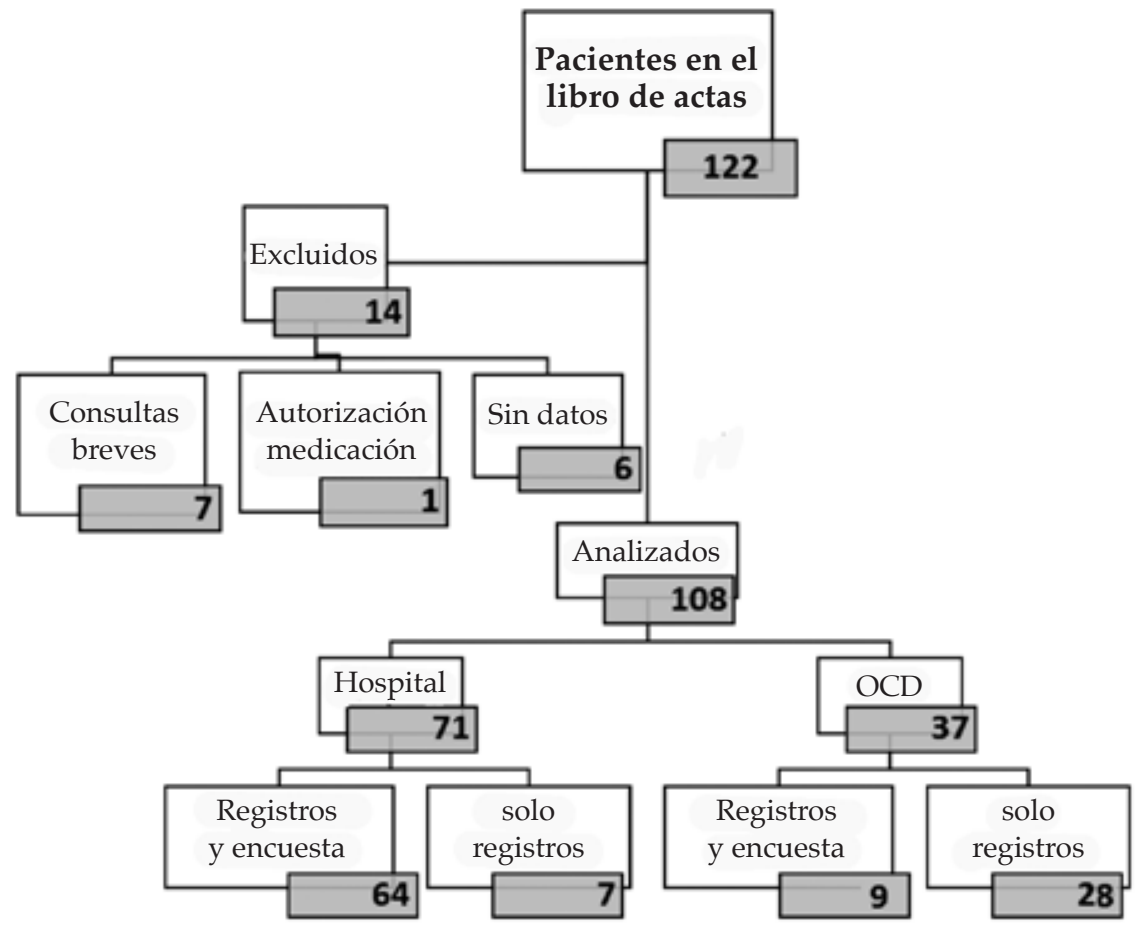




\section{RESULTADOS}

De los 122 casos, 14 fueron excluidos por falta de datos, solicitud aislada de autorización de medicación o consultas breves resueltas individualmente por un miembro del CEC.

El análisis final quedó en 108 casos (106 pacientes), 73 casos con encuesta y registros, y 35 solamente con registros (HC, libro de actas y OCD) (Figura 1). La distribución de casos presentados por año es regular y los datos demográficos de los pacientes se detallan en la Tabla 1.

Los motivos principales más frecuentes de consulta fueron los siguientes (n/\%): adecuación del esfuerzo terapéutico $(46 / 42,6 \%)$, compromiso neurológico grave $(15 / 13,9 \%)$, calidad de vida del paciente $(11 / 10,2 \%)$, conflictos surgidos entre el paciente, la familia y el sistema de salud $(7 / 6,5 \%)$, y rechazo del tratamiento por la familia $(6 / 5,6 \%)$. Todos los motivos principales de consulta se consignan en la Figura 2 y los motivos principales y secundarios agrupados, en la Figura 3 (en Anexo electrónico).

El número de reuniones por caso tuvo una mediana de 1 (rango 1-5); 20 (19\%) fueron de urgencia. Solo en 1 caso el CEC tuvo la iniciativa de dar intervención judicial y en 4 oportunidades recomendó dar intervención a organismos protectores de los derechos de la niñez y la adolescencia.
En la Tabla 2 se puede ver un listado no exhaustivo de las especialidades que participaron en las consultas al CEC (en Anexo electrónico).

En todos los casos se realizó un informe con recomendaciones escritas; sin embargo, solo en $31(28,7 \%)$ dicho documento estaba en la HC. A estos 31 casos se suman $19(17,6 \%)$ donde un profesional de salud realizó una transcripción

TABLA 1. Consultas por año y demografía

\begin{tabular}{lc}
\hline Año & $\begin{array}{c}\text { Consultas } \\
\mathbf{n}(\%)\end{array}$ \\
\hline 2013 & $18(16,7)$ \\
2014 & $21(19,4)$ \\
2015 & $23(21,3)$ \\
2016 & $24(22,2)$ \\
2017 & $22(20,4)$ \\
\hline
\end{tabular}

\begin{tabular}{lc}
\hline $\begin{array}{l}\text { Demografía } \\
\text { Sexo }\end{array}$ & \\
Masculino & $62(58)$ \\
Femenino & $43(41)$ \\
Intersexo & $1(1)$ \\
\hline Edad & \\
Neonato & $2(1,9)$ \\
$1-24$ meses & $42(38,8)$ \\
$2-13$ años & $47(43,5)$ \\
$13-15$ años & $6(5,6)$ \\
$>16$ años & $10(9,3)$ \\
Edad sin datos & $1(0,9)$ \\
\hline
\end{tabular}

n: número.

FIGURA 2 Motivos principales de consulta

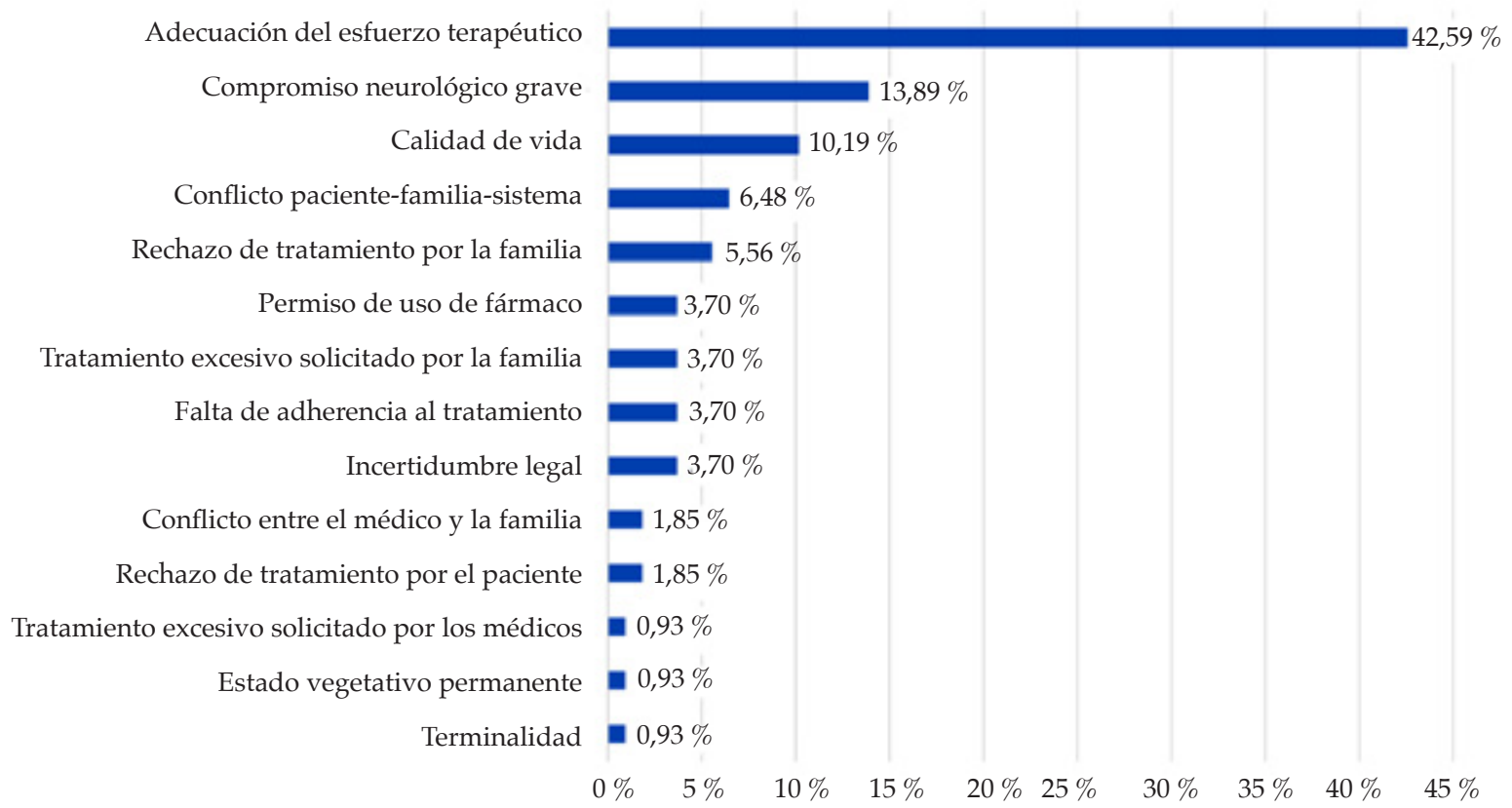


parcial en la HC. Es decir que solo en 50 de los 108 casos (46,3\%) quedó registro en la HC de la consulta realizada al CEC.

\section{Perfil y opiniones de los profesionales entrevistados}

Respondieron 73 profesionales $(69,4 \%), 54$ de sexo femenino $(73,9 \%$ ) y con una media (rango) de edad y antigüedad laboral de 49 (35-63) y 12 (4-40) años respectivamente. Las especialidades, con el área de experiencia de algunas, se detallan en la Tabla 3 (en Anexo electrónico). Los casos no encuestados fueron por imposibilidad de contactar al equipo tratante (29), negativa a ser entrevistados o no recordar el caso (6).

En 46 casos (64\%), los profesionales no modificaron sus opiniones iniciales, en 16 $(22 \%)$, lo hicieron parcialmente y, en $10(14 \%)$, modificaron su opinión. Asimismo, 58 (80\%) afirmaron que la consulta al CEC aumentó sus competencias para abordar casos difíciles, 10 $(14 \%)$ lo consideraron de manera parcial y $5(6 \%)$ que no la aumentaron.

Respecto de si la consulta contribuyó a aclarar las dudas legales, 38 profesionales (52\%) opinaron que contribuyó, $15(21 \%)$ dijeron que la contribución fue parcial, en tanto que 20 (27\%) consideraron que no contribuyó.

Los resultados clasificados como de factibilidad, efectividad y satisfacción se resumen a continuación. Se detallan en forma completa en la Tabla 4.

\section{Factibilidad}

De los encuestados, 63 (86\%) consideraron que las sugerencias del CEC llegaron dentro de un plazo oportuno para la toma de decisiones con el paciente, $60(82 \%)$ consideraron que la consulta al CEC facilitó la adecuada toma de decisiones del equipo de salud y, respecto a la puesta en práctica de las decisiones, $54(74 \%)$ las sintieron facilitadas.

Se pudo evaluar coherencia entre la conducta y la recomendación en 59 casos (54,6 \%). La recomendación se siguió totalmente en 50 (85\%).

\section{Efectividad}

En cuanto a si la recomendación del CEC impactó positivamente en la familia o en el propio paciente, 31 profesionales ( $44 \%$ ) consideraron que sí. La consulta al CEC no empeoró la situación para 68 encuestados $(93 \%)$.

De los 108 casos registrados, se pudo evaluar coherencia en la evolución real y en la esperada en $55(50,9 \%)$, que coincidió en $47(91 \%)$ de los casos de manera total o parcial.

\section{Satisfacción}

Hubo acuerdo con la recomendación brindada por el CEC en 69 (95\%) profesionales. Acerca de la inversión de tiempo en la preparación y ejecución de la consulta, estuvo justificada por el resultado para 71 encuestados $(97 \%)$; la consulta al CEC fue una experiencia globalmente positiva para 70 encuestados $(96 \%)$.

Los comentarios que hubo en $38 / 73$ encuestas (52\%) se reportan agrupados por temas en la Tabla 5 (en Anexo electrónico).

\section{DISCUSIÓN}

Se han encontrado pocos estudios, en adultos o pediátricos, con los cuales contrastar los resultados aquí presentados. En la Tabla 6 se expone una síntesis de los resultados de este trabajo comparados con la literatura hallada. ${ }^{12-24}$

Los indicadores de calidad habitualmente se clasifican como de estructura, proceso y resultado. ${ }^{25}$ En esa lógica, los hallazgos clasificados como FES (factibilidad, efectividad y satisfacción) son de resultados de la consulta ética. Cuantificar los resultados de la consulta ética es dificultoso. Tanto es así, que sigue en discusión si son resultados lo que hay que evaluar o si la actividad consultiva ética se debe evaluar solo en las estructuras y procesos. ${ }^{8,26,27}$ Por eso se puede considerar incorrecto evaluar a un CEC sobre la base de criterios de factibilidad, efectividad y satisfacción. Se puede decir que las mencionadas características no son objetivos del CEC, sino que el objetivo es dar recomendaciones correctas o buenas, fundamentadas en razonamientos éticos sólidos y justos. ${ }^{22}$

Si una recomendación es la más correcta, podría importar poco la factibilidad, la satisfacción, etc. Sin embargo, es fácil imaginar escenarios donde el CEC puede hacer recomendaciones correctas desde el punto de vista ético, pero que el equipo tratante no logra llevar a cabo, no por falencia de la recomendación, sino por fallas del equipo. También podría suceder que recomendaciones éticamente perfectas no sean satisfactorias para el equipo tratante. Por otro lado, un CEC puede hacer razonamientos éticamente correctos, pero llegar a recomendaciones equivocadas si el equipo tratante no ha hecho una evaluación técnicomédica acertada de los diagnósticos, alternativas terapéuticas y probabilidades pronósticas. 
Por eso, no alcanza con hacer recomendaciones correctas, ya que un objetivo principal del CEC es que lo correcto ocurra en el paciente. Si esto no pasa, igualmente se puede atribuir falla al CEC en objetivos centrales de su funcionamiento, como son crear consenso, mediar, manejar conocimiento y actitud inquisidora frente a las posibilidades clínicas.

En la díada CEC-equipo tratante, cuyo propósito es consensuar aquella decisión que mejor respete los valores del paciente y su familia, poco importa en la práctica qué grupo "se equivoca" más. Lo realmente importante es que ambas partes no pierdan el objetivo que los reúne y que siempre se busque la mejora continua.

Desde otra perspectiva, Mercurio ${ }^{2}$ aborda el problema de cuantificar el desempeño de un CEC igualándolo a "satisfacción del grupo tratante y/o la familia", ${ }^{15}$ argumento también defendido por Delany y Hall. ${ }^{28}$ Ya se analizaron algunas debilidades de homologar satisfacción con desempeño, que se suman a las expuestas por otros autores. ${ }^{6}$ Otro riesgo de buscar la satisfacción del grupo tratante es el de convertir al CEC en solo un brazo más de la institución, que ejerza poder de persuasión sobre el paciente y su

TABla 4. Factibilidad, efectividad y satisfacción

\begin{tabular}{|c|c|c|c|c|c|c|}
\hline \multicolumn{7}{|c|}{ Factibilidad } \\
\hline Fuente & Variable & En nada & $\begin{array}{l}\text { Casi } \\
\text { nada }\end{array}$ & Parcialmente & $\begin{array}{l}\text { Casi } \\
\text { totalmente }\end{array}$ & Totalmente \\
\hline Encuesta & $\begin{array}{l}\text { ¿En cuánto la consulta al CEC facilitó la } \\
\text { adecuada toma de decisiones } \\
\text { del equipo de salud? }\end{array}$ & $\begin{array}{c}2 \\
(3 \%)\end{array}$ & $\begin{array}{c}0 \\
(0 \%)\end{array}$ & $\begin{array}{c}11 \\
(15 \%)\end{array}$ & $\begin{array}{c}25 \\
(34 \%)\end{array}$ & $\begin{array}{c}35 \\
(48 \%)\end{array}$ \\
\hline Encuesta & $\begin{array}{l}\text { ¿En cuánto la consulta al CEC facilitó } \\
\text { la puesta en práctica de las decisiones? }\end{array}$ & $\begin{array}{c}1 \\
(1 \%)\end{array}$ & $\begin{array}{c}2 \\
(3 \%)\end{array}$ & $\begin{array}{c}16 \\
(22 \%)\end{array}$ & $\begin{array}{c}24 \\
(33 \%)\end{array}$ & $\begin{array}{c}30 \\
(41 \%)\end{array}$ \\
\hline Encuesta & $\begin{array}{l}\text { ¿En cuánto las recomendaciones del CEC, } \\
\text { ya sean verbales o escritas, le llegaron a tiempc } \\
\text { para la toma de decisiones con el paciente? }\end{array}$ & $\begin{array}{c}3 \\
(4 \%)\end{array}$ & $\begin{array}{c}0 \\
(0 \%)\end{array}$ & $\begin{array}{c}7 \\
(7 \%)\end{array}$ & $\begin{array}{c}14 \\
(14 \%)\end{array}$ & $\begin{array}{c}49 \\
(67 \%)\end{array}$ \\
\hline $\mathrm{HC}$ & Coherencia conductas/recomendación & & $\begin{array}{c}1 \\
(2 \%)\end{array}$ & $\begin{array}{c}8 \\
(13 \%)\end{array}$ & & $\begin{array}{c}50 \\
(85 \%)\end{array}$ \\
\hline
\end{tabular}

\begin{tabular}{|c|c|c|c|c|c|c|c|}
\hline Fuente & Variable & $\begin{array}{c}\text { En } \\
\text { nada }\end{array}$ & $\begin{array}{l}\text { Casi } \\
\text { nada }\end{array}$ & Parcialmente & $\begin{array}{l}\text { Casi } \\
\text { totalmente }\end{array}$ & Totalmente & $\mathbf{N}$ \\
\hline Encuesta & $\begin{array}{l}\text { ¿En cuánto la recomendación del CEC impactó } \\
\text { positivamente en la familia o } \\
\text { en el propio paciente? }\end{array}$ & $\begin{array}{c}9 \\
(13 \%)\end{array}$ & $\begin{array}{c}7 \\
(10 \%)\end{array}$ & $\begin{array}{c}23 \\
(33 \%)\end{array}$ & $\begin{array}{c}14 \\
(20 \%)\end{array}$ & $\begin{array}{c}17 \\
(24 \%)\end{array}$ & 70 \\
\hline Encuesta & La consulta al CEC empeoró la situación & $\begin{array}{c}66 \\
(90 \%)\end{array}$ & $\begin{array}{c}2 \\
(3 \%)\end{array}$ & $\begin{array}{c}3 \\
(4 \%)\end{array}$ & $\begin{array}{c}0 \\
(0 \%)\end{array}$ & $\begin{array}{c}2 \\
(3 \%)\end{array}$ & 73 \\
\hline $\mathrm{HC}$ & Coherencia evolución real/esperada & \multicolumn{2}{|c|}{$\begin{array}{c}5 \\
(9,0 \%)\end{array}$} & $\begin{array}{c}3 \\
(5,5 \%)\end{array}$ & & $\begin{array}{c}47 \\
(85,5 \%)\end{array}$ & 75 \\
\hline
\end{tabular}

\begin{tabular}{llcccccc}
\hline Satisfacción & & & & & \\
\hline Fuente & Variable & $\begin{array}{c}\text { En } \\
\text { nada }\end{array}$ & $\begin{array}{c}\text { Casi } \\
\text { nada }\end{array}$ & Parcialmente & $\begin{array}{c}\text { Casi } \\
\text { totalmente }\end{array}$ & Totalmente & N \\
\hline Encuesta & ¿En cuánto acordó con la recomendación? & 0 & 0 & 4 & 10 & 59 & 73 \\
& & $(0 \%)$ & $(0 \%)$ & $(5 \%)$ & $(14 \%)$ & $(81 \%)$ & 60 \\
Encuesta & La inversión de tiempo en la preparación y & 0 & 1 & 1 & 11 & $(82,2 \%)$ \\
& $\begin{array}{l}\text { ejecución de la consulta al CEC estuvo } \\
\text { justificada por el resultado }\end{array}$ & $(0 \%)$ & $(1,4 \%)$ & $(1,4 \%)$ & $(15 \%)$ & $(3)$ \\
\hline
\end{tabular}

\begin{tabular}{llcccccc}
\hline Factibilidad & & & & & & \\
\hline Fuente & Variable & $\begin{array}{c}\text { En } \\
\text { nada }\end{array}$ & $\begin{array}{c}\text { Casi } \\
\text { nada }\end{array}$ & Parcialmente & $\begin{array}{c}\text { Casi } \\
\text { totalmente }\end{array}$ & Totalmente & N \\
\hline Encuesta & La consulta al CEC fue una experiencia & 0 & 2 & 1 & 8 & 62 & 73 \\
& globalmente positiva & $(0 \%)$ & $(3 \%)$ & $(1 \%)$ & $(11 \%)$ & $(85 \%)$ & \\
\hline
\end{tabular}

HC: historia clínica; CEC: comité de ética clínica. 


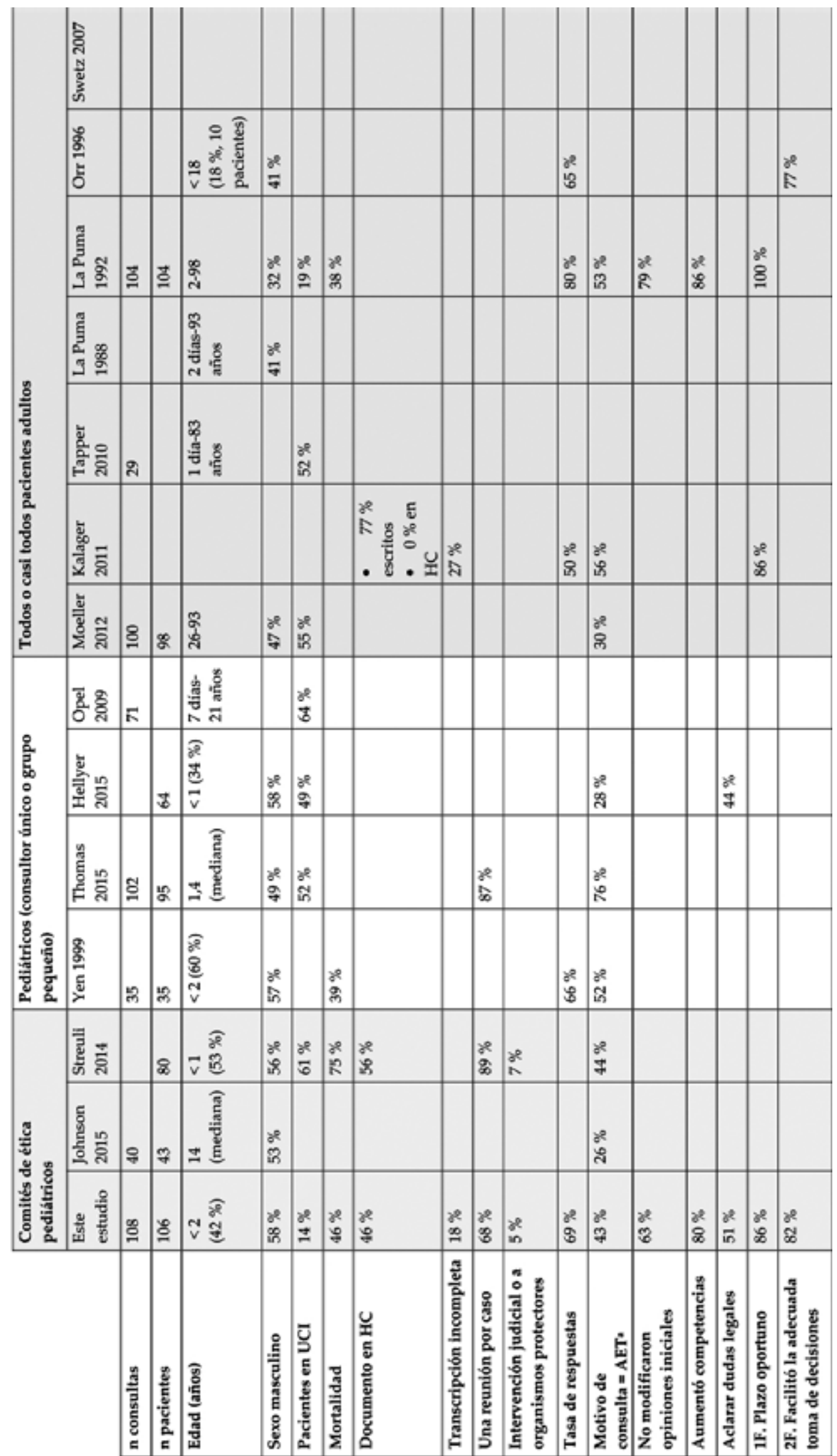



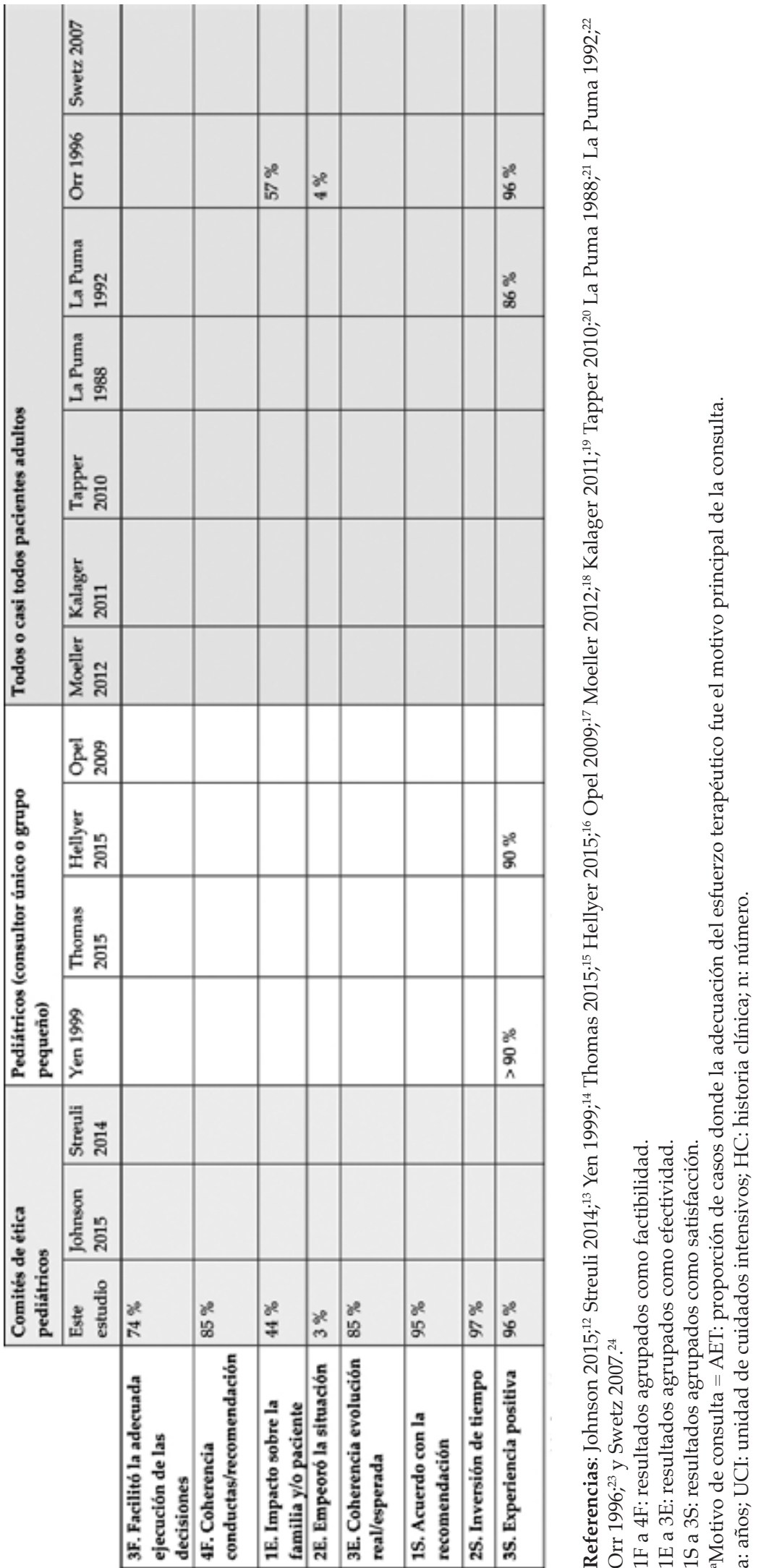
familia, conformando algo similar al imperialismo cultural descripto por DeMichelis. ${ }^{29}$

Otras medidas de efectividad de los CEC propuestas son la disminución de días de internación en unidades de cuidados intensivos (UCI) y el uso de tratamientos de soporte vital en pacientes que no sobreviven, abordada por Schneiderman y cols., en dos estudios. ${ }^{30,31}$ Esta segunda medida de resultado conlleva la sospecha del sesgo referido a veces como el de la "profecía autocumplida". Es lógico pensar que, si se disminuye el soporte vital, consecuentemente disminuirá la supervivencia en UCI. Por lo tanto, no se puede concluir solamente con esos datos que esa disminución haya sido correcta, excesiva o insuficiente.

Ejemplos de posibles profecías autocumplidas dan fundamento a esta objeción e incluyen casos individuales y conductas sistemáticas. Austin y cols. describen una paciente con pronóstico estimado como ominoso, donde el equipo de salud y la familia acordaron la interrupción del soporte vital, solo demorada unos días para permitir que lleguen familiares alejados geográficamente. Esta demora permitió la recuperación completa de la paciente y demostrar que el pronóstico no era acertado. ${ }^{32}$ Green y cols., describen la decisión sistemática del retiro de la oxigenación por membrana extracorpórea por futilidad pulmonar a los 5 a 14 días de asistencia en pacientes con características clínicas similares que posteriormente se comprobó que podían sobrevivir si se mantenía el soporte durante un tiempo más prolongado. ${ }^{33}$ Becker y cols., en su estudio del modelo pronóstico de hemorragia intracraneana, describen cómo la decisión de retiro de soporte vital y el pesimismo influyen en la toma de decisiones más que otros factores objetivos, tales como tamaño de la hemorragia o la escala de Glasgow. ${ }^{34}$ Ferrand y Racine, en un trabajo reciente, analizan las dificultades que enfrenta el equipo de salud para la toma de decisiones basada en el pronóstico, lo que refuerza la necesidad de mantenerse humilde y con mente abierta en las discusiones basadas en estimaciones pronósticas. ${ }^{35}$

En relación con la taxonomía (factibilidad, efectividad y satisfacción), no se pretende que sea universalmente aceptada, ya que su utilización es claramente discutible. Por ejemplo, los ítems de factibilidad pueden ser interpretados como de satisfacción ("las recomendaciones llegaron a tiempo") o efectividad ("facilitó la puesta en práctica", "coherencia entre conductas y recomendación"). Como muchas taxonomías, esta es tan solo un intento de hacer más comprensible el análisis de temas complejos de bordes difusos.

Otras limitaciones de este trabajo surgen de la metodología aplicada. Gran parte de la información obtenida proviene de las respuestas del personal de salud encuestado, las cuales están sujetas a riesgos de sesgos. Individualmente, los entrevistados pueden inclinar sus respuestas hacia la cortesía debido a deseos, conscientes o no, de agradar o por respeto a la institución. En forma opuesta, pueden ocurrir sesgos negativos en las mismas respuestas. No existe manera de descartar o dimensionar hacia qué lado ocurrieron estos y otros sesgos del método de encuestas.

A diferencia de otras investigaciones, ${ }^{15,24,36}$ este estudio abordó la experiencia de los pacientes y sus familias a través de la opinión del equipo tratante. En ningún momento se intentó abordarlas directamente. Aunque siempre se explicita, en las recomendaciones por escrito y en la reunión con el equipo tratante, que el CEC está disponible para cualquier necesidad que pueda manifestar la familia, es muy raro que esta solicitud ocurra. Posiblemente, esto se deba a que, desde sus orígenes, el CEC del Hospital Garrahan se formó como un órgano consultivo del equipo tratante y es a él adonde se dirigen los esfuerzos. En la mayoría de los casos, el equipo tratante tiene un fuerte vínculo con el paciente y su familia, por lo que la intromisión del CEC en esa relación médico-paciente puede ser vista como una desventaja que puede generar efectos adversos o insatisfacción. ${ }^{15,37}$ Cuando el equipo tratante pide la colaboración del CEC para realizar la devolución de los resultados de la reunión a la familia, esta se canaliza mediante la participación de entre 1 y 3 miembros del CEC.

Están descriptas otras metodologías para evaluar el desempeño de los consultores individuales, pequeños grupos o comités para las consultas éticas, que no han sido abordadas en esta investigación, como el análisis de la estructura del CEC o el proceso de realización de la consulta. ${ }^{9,38}$. Las evaluaciones de estructura se enfocan en los componentes, certificaciones y competencias de los miembros del CEC. Otros análisis de procesos son, por ejemplo, el análisis del informe escrito producido en la consulta ética mediante una herramienta holística desarrollado por Pearlman y cols.,${ }^{39}$ o mediante listas de verificación por Flicker y cols. ${ }^{40}$

Teniendo en cuenta las limitaciones y problemas mencionados en este estudio y por otros autores, ${ }^{6,11,12}$ consideramos que los resultados de este trabajo son relevantes. Son el paso inicial en nuestro hospital en la aplicación de herramientas de mejora continua para el fortalecimiento de los procesos utilizados por el CEC.

\section{CONCLUSIONES}

Este trabajo reveló altos niveles de satisfacción (> $95 \%$ ) y moderados de factibilidad $(>74 \%)$ y efectividad $(>85 \%)$. El $80 \%$ de los profesionales 
afirmó que la consulta al CEC aumentó sus competencias para abordar casos difíciles y el $51 \%$, que contribuyó a aclarar dudas legales. Sin embargo, solo el $44 \%$ opinó que la consulta tuvo un impacto positivo en la familia y el paciente. Los hallazgos del estudio impulsaron mejoras en el desarrollo de las consultas al CEC, especialmente en la documentación, la comunicación en el proceso de consulta y en la reevaluación del contacto directo de los padres y pacientes con el CEC.

\section{REFERENCIAS}

1. Rivera Montero R. Comités hospitalarios de bioética. En: Sepúlveda JA. Bioética en pediatría. México, D.F.: Manual Moderno; 2014.Pág.13.

2. Mercurio MR. Pediatric ethics committes. En: Miller G(ed). Pediatric Bioethics. Cambridge: Cambridge University Press; 2010.Págs.87-110.

3. Lo B. Behind closed doors. Promises and pitfalls of ethics committees. N Engl J Med. 1987; 317(1):46-50.

4. Craig JM, May T. Evaluating the outcomes of ethics consultation. J Clin Ethics. 2006; 17(2):168-80.

5. Williamson L. Empirical assessments of clinical ethics services: implications for clinical ethics committees. Clin Ethics. 2007; 2(4):187-92.

6. Slowther AM, Hope T. Clinical ethics committees. BMJ. 2000; 321(7262):649-50.

7. Au SS, Couillard P, des Ordons AR, Fiest KM, et al. Outcomes of Ethics Consultations in Adult ICUs: A Systematic Review and Meta-Analysis. Crit Care Med. 2018; 46(5):799-808.

8. Aulisio MP, Arnold RM, Youngner SJ. Health care ethics consultation: nature, goals, and competencies. A position paper from the Society for Health and Human ValuesSociety for Bioethics Consultation Task Force on Standards for Bioethics Consultation. Ann Intern Med. 2000; 133(1):5969.

9. University of Toronto Joint Centre for Bioethics. Evaluating Excellence in Bioethics: a value for investment project, 2010. In University of Toronto Joint Centre for Bioethics Review. 2011:67-104. [Acceso: 5 de agosto de 2021]. Disponible en: http: / / 128.100.72.105/about/documents/FINAL_UofT_ JCB_Review.pdf

10. Williamson L, McLean S, Connell J. Clinical ethics committees in the United Kingdom: towards evaluation. Med Law Int. 2007; 8(3):221-38.

11. Repenshek M. Continuous Quality Improvement Initiatives in Ethics: A Proposed Communication Tool. Health Care Ethics USA. 2012.Págs.2-12.

12. Johnson LM, Church CL, Metzger M, Baker JN. Ethics consultation in pediatrics: long-term experience from a pediatric oncology center. Am J Bioeth. 2015; 15(5):3-17.

13. Streuli JC, Staubli G, Pfändler-Poletti M, BaumannHölzle R, Ersch J. Five-year experience of clinical ethics consultations in a pediatric teaching hospital. Eur J Pediatr. 2014; 173(5):629-36.

14. Yen BM, Schneiderman LJ. Impact of pediatric ethics consultations on patients, families, social workers, and physicians. J Perinatol. 1999; 19(5):373-8.

15. Thomas SM, Ford PJ, Weise KL, Worley S, Kodish E. Notjust little adults: a review of 102 paediatric ethics consultations. Acta Paediatr. 2015; 104(5):529-34.

16. Henriksen Hellyer J, Schiltz B, Moon W, Grafelman M, et al. Pediatric clinical ethics consultations at an academic medical center: does one size fit all? Am J Bioeth. 2015; 15(5):20-4.

17. OpelDJ, Wilfond BS, Brownstein D, Diekema DS, Pearlman $\mathrm{RA}$. Characterisation of organisational issues in paediatric clinical ethics consultation: a qualitative study. JMed Ethics. 2009; 35(8):477-82.
18. Moeller JR, Albanese TH, Garchar K, Aultman JM, et al. Functions and outcomes of a clinical medical ethics committee: a review of 100 consults. HEC Forum. 2012; 24(2):99-114.

19. Kalager G, Førde R, Pedersen R. Is the discussion of patient cases in clinical ethics-committees useful? Tidsskr Nor Laegeforen. 2011; 131(2):118-21.

20. Tapper EB, Vercler CJ, Cruze D, Sexson W. Ethics consultation at a large urban public teaching hospital. Mayo Clin Proc. 2010; 85(5):433-8.

21. La Puma J, Stocking CB, Silverstein MD, DiMartini A, Siegler M. An ethics consultation service in a teaching hospital. Utilization and evaluation. JAMA. 1988; 260(6):808-11.

22. La Puma J, Stocking CB, Darling CM, Siegler M. Community hospital ethics consultation: evaluation and comparison with a university hospital service. Am JMed.1992;92(4):34651.

23. Orr RD, Morton KR, deLeon DM, Fals JC. Evaluation of an ethics consultation service: patient and family perspective. Am J Med. 1996; 101(2):135-41.

24. Swetz KM, Crowley ME, Hook C, Mueller PS. Report of 255 clinical ethics consultations and review of the literature. Mayo Clin Proc. 2007; 82(6):686-91.

25. Donabedian A. The quality of care. How can it be assessed? JAMA. 1988; 260(12):1743-8.

26. Schildmann J, Nadolny S, Haltaufderheide J, Vollmann J, et al. Measuring Outcomes of Ethics Consultation: Empirical and Ethical Challenges. Crit Care Med. 2019; 47(1):e67-8.

27. Au SS. The author replies. Crit Care Med. 2019; 47(1):e68.

28. Delany C, Hall G. 'I just love these sessions'. Should physician satisfaction matter in clinical ethics consultations? Clin Ethics. 2012; 7(3):116-21.

29. DeMichelis C. The Best Interests Standard as a Logic of Empire: Unpacking the Political Dimensions of Parental Refusal. Am J Bioeth. 2018; 18(8):83-5.

30. Schneiderman LJ, Gilmer T, Teetzel HD. Impact of ethics consultations in the intensive care setting: a randomized, controlled trial. Crit Care Med. 2000; 28(12):3920-4.

31. Schneiderman LJ, Gilmer T, Teetzel HD, Dugan DO, et al. Effect of ethics consultations on nonbeneficial lifesustaining treatments in the intensive care setting: a randomized controlled trial. JAMA. 2003; 290(9):1166-72.

32. Austin EB, Hobbs H, Crouse BA, Lobos AT. A case report of full recovery from severe cerebral edema secondary to acetaminophen-induced hepatotoxicity in a13 year old girl. BMC Pediatr. 2018; 18(1):247.

33. Green TP, Moler FW, Goodman DM. Probability of survival after prolonged extracorporeal membrane oxygenation in pediatric patients with acute respiratory failure. Extracorporeal Life Support Organization. Crit Care Med. 1995; 23(6):1132-9.

34. Becker KJ, Baxter AB, Cohen WA, Bybee HM, et al. Withdrawal of support in intracerebral hemorrhage may lead to self-fulfilling prophecies. Neurology. 2001;56(6):76672.

35. Ferrand A, Racine E. Can Clinicians Be Objective? Inherent Challenges in Using Decision-Making Tools in Cases of Entrenched Disagreements. Am J Bioeth. 2018; 18(8):80-2.

36. McClung JA, Kamer RS, DeLuca M, Barber HJ. Evaluation of a medical ethics consultation service: opinions of patients and health care providers. Am I Med. 1996; 100(4):456-60.

37. Matheny Antommaria AH. Intensified Conflict Instead of Closure: Clinical Ethics Consultants' Recommendations' Potential to Exacerbate Ethical Conflicts. Am J Bioeth. 2015; 15(1):52-4.

38. Larcher V, Slowther AM, Watson AR, UK Clinical Ethics Network. Core competencies for clinical ethics committees. Clin Med (Lond). 2010; 10(1):30-3.

39. Pearlman RA, Foglia MB, Fox E, Cohen JH, et al. Ethics Consultation Quality Assessment Tool: A Novel Method for Assessing the Quality of Ethics Case Consultations Based on Written Records. Am J Bioeth. 2016; 16(3):3-14.

40. Flicker LS, Rose SL, Eves MM, Flamm AL, et al. Developing and testing a checklist to enhance quality in clinical ethics consultation. J Clin Ethics. 2014; 25(4):281-90. 


\section{ANEXO}

FIGURA 3. Motivos principales y secundarios agrupados

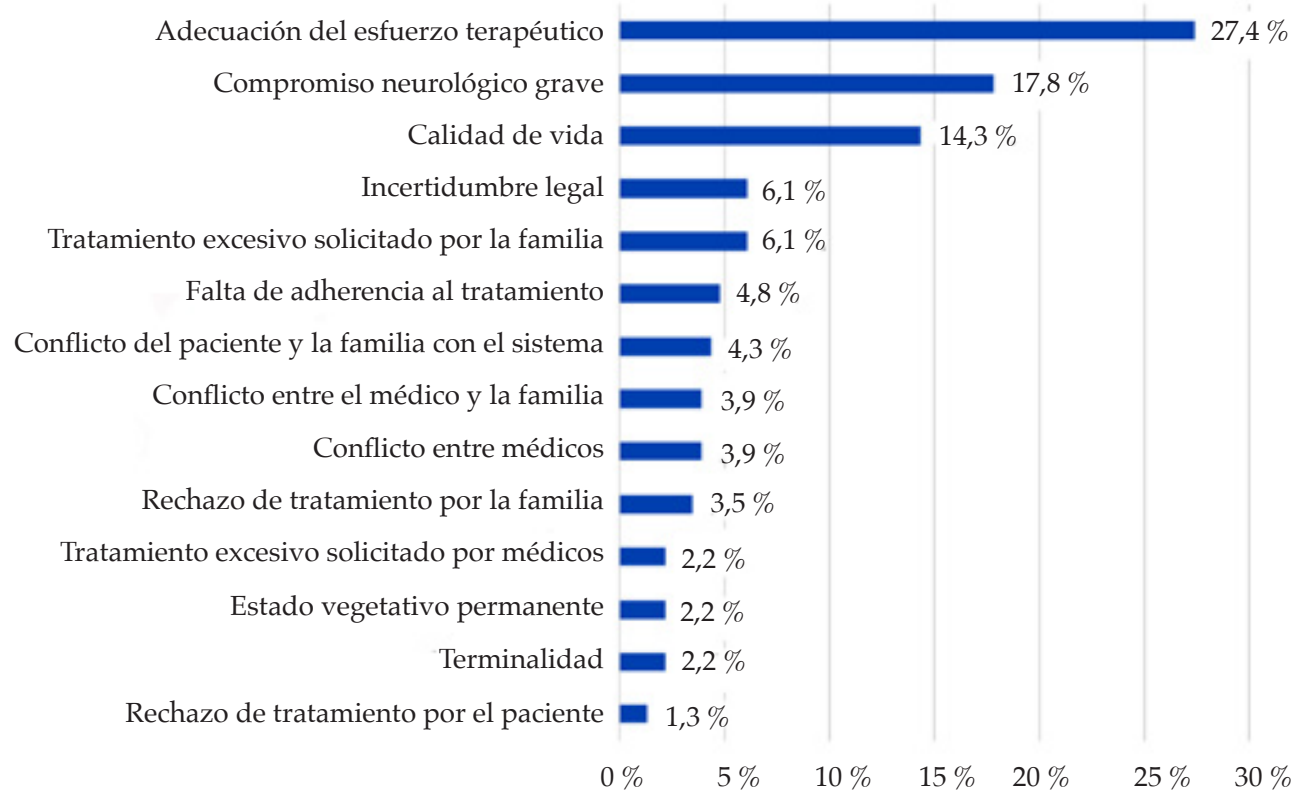

Uno o más motivos por caso. Total $=230$ motivos en 108 casos.

TABLA 2 Especialidades participantes en las consultas

\begin{tabular}{|c|c|c|c|c|c|}
\hline Especialidad & $\mathbf{n}$ & $\%$ & Especialidad & $\mathbf{n}$ & $\%$ \\
\hline 1. Salud mental & 34 & 11,4 & 19. Ortopedia y traumatología & 5 & 1,7 \\
\hline 2. Cuidados paliativos & 32 & 10,7 & 20. Neonatología & 5 & 1,7 \\
\hline 3. Neurología & 32 & 10,7 & 21. Bioética & 5 & 1,7 \\
\hline 4. Servicio social & 20 & 6,7 & 22. Cirugía cardiovascular & 3 & 1 \\
\hline 5. Cardiología & 16 & 5,4 & 23. Cirugía plástica y quemados & 3 & 1 \\
\hline 6. Hematooncología & 16 & 5,4 & 24. Dermatología & 3 & 1 \\
\hline 7. Neurocirugía & 15 & 5 & 25. Errores congénitos del metabolismo & 3 & 1 \\
\hline 8. Nefrología & 13 & 4,3 & 26. Patología espinal & 3 & 1 \\
\hline 9. Genética & 12 & 4 & 27. Terapia intensiva & 3 & 1 \\
\hline 10. Gastroenterología & 8 & 2,7 & 28. Trasplante de médula ósea & 3 & 1 \\
\hline 11. Neumonología & 8 & 2,7 & 29. Radiología e intervencionismo & 3 & 1 \\
\hline 12. Pediatría & 8 & 2,7 & 30. Clínicas interdisciplinarias & 2 & 0,7 \\
\hline 13. Cirugía general & 7 & 2,3 & 31. Endoscopia respiratoria & 2 & 0,7 \\
\hline 14. Hepatología & 6 & 2 & 32. Hemoterapia & 2 & 0,7 \\
\hline 15. Crecimiento y desarrollo & 5 & 1,7 & 33. Infectología & 2 & 0,7 \\
\hline 16. Endocrinología & 5 & 1,7 & 34. Adolescencia & 2 & 0,7 \\
\hline 17. Nutrición & 5 & 1,7 & 35. Urología & 1 & 0,3 \\
\hline \multirow[t]{2}{*}{ 18. Oftalmología } & 5 & 1,7 & 36. Diagnóstico por imágenes & 1 & 0,3 \\
\hline & & & Total & 298 & 100 \\
\hline
\end{tabular}

n: número. 
TABLA 3. Especialidad profesional y área de experiencia* de los entrevistados

\begin{tabular}{|c|c|c|c|c|c|}
\hline Especialidad y área de experiencia & $\mathbf{n}$ & $\%$ & Especialidad y área de experiencia & $\mathbf{n}$ & $\%$ \\
\hline Clínica pediátrica & 22 & 30,14 & Cuidados paliativos & 3 & 4,11 \\
\hline Clínica general & 13 & 17,81 & Neurología & 3 & 4,11 \\
\hline Internación & 6 & 8,22 & Enfermedad neuromuscular & 2 & 2,74 \\
\hline Emergencia & 2 & 2,74 & Epilepsia & 1 & 1,37 \\
\hline Condiciones crónicas complejas & 1 & 1,37 & Crecimiento y desarrollo & 2 & 2,74 \\
\hline Terapia intensiva & 13 & 17,81 & Trasplante hepático & 2 & 2,74 \\
\hline Terapia intensiva general & 12 & 16,44 & Oftalmología & 2 & 2,74 \\
\hline Inmunocomprometidos & 1 & 1,37 & Errores congénitos del metabolismo & 2 & 2,74 \\
\hline Nefrología & 7 & 9,59 & Servicio social & 1 & 1,37 \\
\hline Nefrología & 4 & 5,48 & Quemados & 1 & 1,37 \\
\hline Trasplante renal & 3 & 4,11 & Neonatología & 1 & 1,37 \\
\hline Hematooncología & 7 & 9,59 & Radiología & 1 & 1,37 \\
\hline Hematología no maligna & 1 & 1,37 & Radiología intervencionista & 1 & 1,37 \\
\hline Oncología & 6 & 8,22 & Kinesiología & 1 & 1,37 \\
\hline Salud mental & 5 & 6,85 & Kinesiología pediátrica & 1 & 1,37 \\
\hline Psicología & 2 & 2,74 & & & \\
\hline Psiquiatría & 2 & 2,74 & & & \\
\hline \multirow[t]{2}{*}{ Oncología } & 1 & 1,37 & & & \\
\hline & & & Total general & 73 & 100 \\
\hline
\end{tabular}

n: número

*Área de experiencia según lo manifestado por el encuestado.

TABLA 5. Comentarios agrupados por temáticas

\begin{tabular}{lcc}
\hline Temas & n & $\%$ \\
\hline Valora positivamente la actividad & 10 & 13,7 \\
Mayor visibilidad de las recomendaciones en la historia clínica & 7 & 9,6 \\
Recomendaciones solo verbales; las escritas llegaron tarde o no llegaron & 3 & 4,1 \\
Insatisfacción con el CEC & 3 & 4,1 \\
Deseo de seguimiento del caso & 2 \\
Recomendaciones más expeditivas & 2 & 2,7 \\
Proponen al CEC como actividad educativa para médicos jóvenes y residentes & 2 \\
Desconfianza de imparcialidad & 1 & 1,7 \\
Queja porque se dedica a responder cómo y no qué & 1 \\
Más objetividad. "Al poner imágenes durante la presentación, se vuelve menos objetivo el caso. & 1 \\
Genera mayor impacto para quienes no vienen viendo al paciente asiduamente, & 1,4 \\
y hay mucha diferencia entre quienes lo conocen y quienes no". & 1 \\
Más subjetividad. “Yo sugeriría que se viera al paciente previo a la toma de decisiones para evitar & 1,4 \\
la despersonalización del paciente. Debería el CEC conocerlos, conocer al paciente y al equipo tratante”. & 1 \\
Mayor participación del CEC en la comunicación de las decisiones & 1,4 \\
Se sintió subestimado por un miembro del CEC & 1 \\
\hline
\end{tabular}

CEC: comité de ética clínica; n: número. 
\begin{tabular}{l|l} 
Hospital de Pediatría S.A.M.I.C."Prof. Dr. Juan P. Garrahan" & 2018 \\
Estudio sobre los resultados de las recomendaciones del CEC & Caso №
\end{tabular}

Sexo: Edad: Antigüedad profesional:

Especialidad principal:

Área de experiencia:

\section{Marque el o los motivos de consulta al Comité de Ética Clínica (CEC)}

(Puede marcar varios y coloque un " 1 " en el principal)

$\square$ Adecuación esfuerzo terapéutico

$\square$ Incertidumbre legal (deber de denunciar, riesgo de cometer mala praxis, etc.)

$\square$ Falta de adherencia al tratamiento

$\square$ Calidad de vida

$\square$ Terminalidad (expectativa de vida $<6$ meses)

$\square$ Compromiso neurológico grave

Estado vegetativo permanente $\square \quad$ Tratamiento excesivo solicitado por médicos

$\square$ Tratamiento excesivo solicitado por familia

$\square$ Rechazo de tratamiento por la familia

- Rechazo de tratamiento por paciente

- Conflicto entre médicos del equipo

$\square$ Conflicto entre equipo médico y familia

$\square$ Conflicto entre paciente-familia y sistema de salud (hospital, obra social, prepaga, etc.)

$\square$ Otro/s (anótelo/s en el reverso)

\section{Marque con una $\mathrm{X}$ la puntuación que considere más acorde en su opinión}

\begin{tabular}{|c|c|c|c|c|c|}
\hline 1. En nada, 2. Casi nada, 3. Parcialmente, 4. Casi totalmente, 5. Totalmente & 1 & 2 & 3 & 4 & 5 \\
\hline \multicolumn{6}{|l|}{ ¿En cuánto acordó con la recomendación? } \\
\hline \multicolumn{6}{|l|}{$\begin{array}{l}\text { ¿En cuánto la consulta CEC facilitó la adecuada toma de } \\
\text { decisiones del equipo de salud? }\end{array}$} \\
\hline \multicolumn{6}{|l|}{$\begin{array}{l}\text { ¿En cuánto la consulta al CEC facilitó la puesta en práctica de } \\
\text { las decisiones? }\end{array}$} \\
\hline \multicolumn{6}{|l|}{$\begin{array}{l}\text { ¿En cuánto las recomendaciones del CEC, ya sean verbales o } \\
\text { escritas, le llegaron a tiempo para la toma de decisiones con } \\
\text { el paciente? }\end{array}$} \\
\hline \multicolumn{6}{|l|}{$\begin{array}{l}\text { ¿En qué medida la consulta al CEC modificó sus opiniones } \\
\text { respecto del caso? }\end{array}$} \\
\hline $\begin{array}{l}\text { ¿En cuánto la recomendación del CEC impactó en las familias } \\
\text { o en el propio paciente en forma positiva? }\end{array}$ & & & & & \\
\hline
\end{tabular}

\begin{tabular}{|c|c|c|c|c|c|}
\hline $\begin{array}{l}\text { 1. Nada de acuerdo, 2. Poco de acuerdo, 3. Ni acuerdo ni desacuerdo, } 4 . \\
\text { Muy de acuerdo, 5. Totalmente de acuerdo }\end{array}$ & 1 & 2 & 3 & 4 & 5 \\
\hline $\begin{array}{l}\text { La inversión de tiempo en la preparación y ejecución de la } \\
\text { consulta al CEC estuvo justificada por el resultado }\end{array}$ & & & & & \\
\hline La consulta al CEC empeoró la situación & & & & & \\
\hline La consulta al CEC fue una experiencia globalmente positiva & & & & & \\
\hline La consulta al CEC contribuyó a aclarar dudas legales & & & & & \\
\hline $\begin{array}{l}\text { La consulta al CEC aumentó en algo sus competencias para } \\
\text { abordar casos difíciles }\end{array}$ & & & & & \\
\hline Comentarios en el reverso & & & & & \\
\hline
\end{tabular}




\section{Observaciones:}


Operacionalización de las variables recabadas desde el libro de actas

\begin{tabular}{|c|c|c|}
\hline Variable & Tipo & Definición operacional \\
\hline Fecha inicial de presentación & Cuantitativa discreta & Fecha en libro de actas \\
\hline Motivo de presentación & Categórica nominal & Explícito/implícito \\
\hline Categoría diagnóstica & Categórica nominal & Especialidad que tuvo la iniciativa en la consulta \\
\hline Otras especialidades relevantes & Categórica nominal & Presentes en la atención del caso \\
\hline $\begin{array}{l}\text { Categorización del origen del problema } \\
\text { ético o en la toma de decisiones }\end{array}$ & Categórica nominal & Lista de opciones más texto libre \\
\hline Número de reuniones del CEC & Cuantitativa discreta & Recuento en libro de actas \\
\hline ¿Caso de resolución urgente? & $\begin{array}{l}\text { Categórica nominal } \\
\text { dicotómica }\end{array}$ & $\begin{array}{l}\text { Aquel en el que se solicita/debe tener una decisión } \\
\text { dentro de un plazo perentorio, en menos de } 7 \text { días: } \\
\text { Sí/no }\end{array}$ \\
\hline ¿Intervención judicial? & $\begin{array}{l}\text { Categórica nominal } \\
\text { dicotómica }\end{array}$ & $\begin{array}{l}\text { Entre las medidas sugeridas en el informe, } \\
\text { ¿se sugiere intervención judicial?: } \\
\text { Sí/no }\end{array}$ \\
\hline $\begin{array}{l}\text { ¿Intervención de otra entidad externa } \\
\text { de protección de los derechos de } \\
\text { la niñez y la adolescencia? }\end{array}$ & $\begin{array}{l}\text { Categórica nominal } \\
\text { dicotómica }\end{array}$ & $\begin{array}{l}\text { Entre las medidas sugeridas en el informe, ¿se sugiere } \\
\text { intervención de otra entidad externa de protección de } \\
\text { los derechos de la niñez y la adolescencia?: } \\
\text { Sí/no }\end{array}$ \\
\hline
\end{tabular}

Operacionalización de las variables recabadas desde la historia clínica

\begin{tabular}{|c|c|c|}
\hline Variable & Tipo & Definición operacional \\
\hline ¿Existe el documento de recomendación? & $\begin{array}{l}\text { Categórica nominal } \\
\text { dicotómica }\end{array}$ & Sí/no \\
\hline $\begin{array}{l}\text { Si no está el documento de recomendación, } \\
\text { ¿existe transcripción en la evolución? }\end{array}$ & $\begin{array}{l}\text { Categórica nominal } \\
\text { dicotómica }\end{array}$ & Sí/no \\
\hline Transcripción & $\begin{array}{l}\text { Categórica nominal } \\
\text { dicotómica }\end{array}$ & Total/parcial \\
\hline Evolución & Categórica nominal & Vivo/muerto/desconocido \\
\hline Fecha de consulta al CEC & Cuantitativa discreta & Fecha según historia clínica \\
\hline Fecha del último registro en la historia clínica & Cuantitativa discreta & Fecha de última evolución en la historia clínica \\
\hline Tiempo de seguimiento & Cuantitativa continua & Fecha última registro - fecha consulta \\
\hline Fecha de óbito & Cuantitativa discreta & Fecha según historia clínica \\
\hline Tiempo de supervivencia & Cuantitativa continua & Fecha óbito y fecha consulta \\
\hline Coherencia conductas/recomendación & Categórica ordinal & $\begin{array}{l}\text { En las conductas registradas en la historia clínica, } \\
\text { la recomendación se siguió } \\
\text { Escala de Likert* }^{*}\end{array}$ \\
\hline Coherencia evolución real/esperada & Categórica ordinal & $\begin{array}{l}\text { La evolución del paciente concuerda con la } \\
\text { presumida por el documento de recomendación } \\
\text { Escala de Likert* }^{*}\end{array}$ \\
\hline
\end{tabular}

*Descriptores de 1 a 3: En nada, parcialmente, totalmente.

¿Cuál fue o fueron los motivos principales de consulta al CEC? (Puede aplicar más de una categoría por caso).

El entrevistado tendrá a la vista las mismas opciones ejemplificadas en "Categorización del origen del problema ético o en la toma de decisiones" más un campo libre para especificar y describir otras no previstas en los ejemplos.

Las siguientes variables serán calificadas subjetivamente por el entrevistado en respuesta al planteo "En su opinión..." mediante una escala de Likert de 5 grados con los siguientes descriptores según corresponda:

1) Nada de acuerdo, 2) poco de acuerdo, 3) ni acuerdo ni desacuerdo, 4) muy de acuerdo, 5) totalmente de acuerdo.

1) En nada, 2) casi nada, 3) parcialmente, 4) casi totalmente, 5) totalmente. 
Operacionalización de las variables recabadas en las entrevistas

\begin{tabular}{|c|c|c|}
\hline Variable & Tipo & Definición operacional \\
\hline Sexo & Categórica nominal dicotómica & Masculino/femenino \\
\hline Edad & Cuantitativa discreta & Años \\
\hline Antigüedad profesional & Cuantitativa discreta & Años \\
\hline Especialidad principal & Categórica nominal & Pediatría o subespecialidad \\
\hline Área de experiencia & Categórica nominal & Dentro de pediatría o subespecialidad \\
\hline Motivos principales de consulta & Categórica nominal & $\begin{array}{l}\text { Selección múltiple de } 1 \text { o más opciones, } \\
\text { señalando el principal + texto libre opcional }\end{array}$ \\
\hline Inversión de tiempo & Categórica ordinal & $\begin{array}{l}\text { "La inversión de tiempo en la preparación y ejecución de la } \\
\text { de la consulta al CEC estuvo justificada por el resultado". } \\
\text { Escala de Likert* }\end{array}$ \\
\hline Plazo oportuno & Categórica ordinal & $\begin{array}{l}\text { "El dictamen/sugerencias del CEC, ya sea verbal o por } \\
\text { escrito, le llegó dentro en un plazo oportuno para la } \\
\text { toma de decisiones con el paciente". } \\
\text { Escala de Likert\# }\end{array}$ \\
\hline Acuerdo con la recomendación & Categórica ordinal & $\begin{array}{l}\text { "Acuerdo con la recomendación". } \\
\text { Escala de Likert\# }\end{array}$ \\
\hline Facilitó la adecuada toma de decisiones & Categórica ordinal & $\begin{array}{l}\text { "La consulta al CEC facilitó la adecuada toma de } \\
\text { decisiones del equipo de salud". } \\
\text { Escala de Likert\# }\end{array}$ \\
\hline $\begin{array}{l}\text { Facilitó la adecuada ejecución de } \\
\text { las decisiones }\end{array}$ & Categórica ordinal & $\begin{array}{l}\text { "La consulta al CEC facilitó la adecuada ejecución de } \\
\text { las decisiones del equipo de salud (ya sean decisiones } \\
\text { previas a la consulta al CEC o decisiones concordantes o } \\
\text { diferentes a las sugeridas por el CEC)". } \\
\text { Escala de Likert* }\end{array}$ \\
\hline Modificó sus opiniones & Categórica ordinal & $\begin{array}{l}\text { "Medida en que la consulta al CEC modificó sus } \\
\text { opiniones respecto del caso". } \\
\text { Escala de Likert* }\end{array}$ \\
\hline Empeoró la situación & Categórica ordinal & $\begin{array}{l}\text { "La consulta al CEC empeoró la situación". } \\
\text { Escala de Likert* }\end{array}$ \\
\hline Experiencia positiva & Categórica ordinal & $\begin{array}{l}\text { "La consulta al CEC fue una experiencia } \\
\text { globalmente positiva". } \\
\text { Escala de Likert\# }\end{array}$ \\
\hline Dudas legales & Categórica ordinal & $\begin{array}{l}\text { "La consulta al CEC contribuyó a aclarar sus dudas legales". } \\
\text { Escala de Likert* }\end{array}$ \\
\hline Competencias & Categórica ordinal & $\begin{array}{l}\text { "La consulta al CEC aumentó en algo sus competencias } \\
\text { en abordar casos difíciles". } \\
\text { Escala de Likert" }\end{array}$ \\
\hline
\end{tabular}

CEC: comité de ética clínica.

\#Descriptores de 1 a 5: 1) Nada de acuerdo, 2) poco de acuerdo, 3) ni acuerdo ni desacuerdo, 4) muy de acuerdo, 5) totalmente de acuerdo

*Descriptores de 1 a 5: 1) En nada, 2) casi nada, 3) parcialmente, 4) casi totalmente, 5) totalmente

A los efectos del análisis, se categorizaron las respuestas en las escalas Likert como positivas y negativas las 2 categorías de cada extremo, y parcial la respuesta o categoría central. 